\title{
Dynamics of Quintom and Hessence Energies in Loop Quantum Cosmology
}

\author{
Hao Wei* \\ Department of Physics and Tsinghua Center for Astrophysics, \\ Tsinghua University, Beijing 100084, China \\ Shuang Nan Zhang \\ Department of Physics and Tsinghua Center for Astrophysics, \\ Tsinghua University, Beijing 100084, China \\ Key Laboratory of Particle Astrophysics, Institute of High Energy Physics, \\ Chinese Academy of Sciences, Beijing 100049, China \\ Physics Department, University of Alabama in Huntsville, Huntsville, AL 35899, USA
}

\begin{abstract}
In the present work, we investigate the universe dominated by quintom or hessence energies in Loop Quantum Cosmology (LQC). Interestingly enough, we find that there are some stable attractors in these two cases. In the case of quintom, all stable attractors have the feature of decelerated expansion. In the case of hessence, most of stable attractors have the feature of decelerated expansion while one stable attractor can have decelerated or accelerated expansion depend on the model parameter. In all cases, the equation-of-state parameter $(\mathrm{EoS})$ of all stable attractors are larger than -1 and there is no singularity in the finite future. These results are different from the dynamics of phantom in LQC, or the ones of phantom, quintom and hessence in classical Einstein gravity.
\end{abstract}

PACS numbers: 95.36.+x, 04.60.Pp, 98.80.-k 


\section{INTRODUCTION}

Dark energy [1] has been one of the most active fields in modern cosmology since the discovery of accelerated expansion of our universe [2, 3, 4, 4, 5, 6, 7, 8]. In the observational cosmology of dark energy, equation-of-state parameter $(\mathrm{EoS}) w_{d e} \equiv p_{d e} / \rho_{d e}$ plays an important role, where $p_{d e}$ and $\rho_{d e}$ are the pressure and energy density of dark energy respectively. Recently, evidence for $w_{d e}(z)<-1$ at redshift $z<0.2 \sim 0.3$ has been found by fitting observational data (see [9, 10, 11, 12, 13, 14, 15, 16] for examples). In addition, many best-fits of the present value of $w_{d e}$ are less than -1 in various data fittings with different parameterizations (see [17] for a recent review). The present data seem to slightly favor an evolving dark energy with $w_{d e}$ being below -1 around present epoch from $w_{d e}>-1$ in the near past [10]. Obviously, the EoS cannot cross the so-called phantom divide $w_{d e}=-1$ for quintessence or phantom alone. Some efforts have been made to build dark energy model whose EoS can cross the phantom divide (see for examples [10, 18, 19, 20, 21, 22, 23, 24, 25, 26, 27, 28, 29, 30, 31, 32, 33, 34, 35] and references therein).

In [10], Feng, Wang and Zhang proposed a so-called quintom model which is a hybrid of quintessence and phantom (thus the name quintom). It is one of the simplest modeles whose EoS can cross the phantom divide. The cosmological evolution of the quintom dark energy was studied in [23, 24]. Perturbations of the quintom dark energy were investigated in [36, 37]; and it is found that the quintom model is stable when EoS crosses -1, in contrast to many dark energy models whose EoS can cross the phantom divide [28]. Other works concerning quintom also include [31] for examples. In [18], by a new view of quintom dark energy, one of us (H.W.) and his collaborators proposed a novel non-canonical complex scalar field, which was named "hessence", to play the role of quintom. In the hessence model, the phantom-like role is played by the so-called internal motion $\dot{\theta}$, where $\theta$ is the internal degree of freedom of hessence. The transition from $w>-1$ to $w<-1$ or vice versa is also possible in the hessence model [18]. The cosmological evolution of the hessence dark energy was studied in [19] and then was extended to the more general cases in [20]. The $w-w^{\prime}$ analysis of hessence dark energy was performed in [21]. In [30], the method to reconstruct hessence dark energy was proposed. We will briefly review the main points of quintom and hessence energies in Sec. II

In fact, many works by now are considered in the framework of classical Einstein gravity. However, it is commonly believed that gravity should also be quantized, like other fundamental forces. As well-known, for many years, the string theory is the only promising candidate for quantum gravity. In the recent decade, however, the Loop Quantum Gravity (LQG) (see e.g. 38, 39, 40, 41] for reviews) has became a competitive alternative to the string theory. LQG is a leading background independent, non-perturbative approach to quantum gravity. At the quantum level, the classical spacetime continuum is replaced by a discrete quantum geometry and the operators corresponding to geometrical quantities have discrete eigenvalues.

Loop Quantum Cosmology (LQC) (see e.g. [42, 43, 44] for reviews) restricts the analysis of LQG to the homogeneous and isotropic spacetimes. Recent investigations have shown that the discrete quantum dynamics can be very well approximated by an effective modified Friedmann dynamics [45, 46, 47]. There are two types of modification to the Friedmann equation due to loop quantum effects [44, 54]. The first one is based on the modification to the behavior of inverse scale factor operator below a critical scale factor $a_{*}$. So far, most of the LQC literature has used this one. Many interesting results have been found, for instance, the replacement of the classical big bang by a quantum bounce with desirable features [43, 45, 48], avoidance of many singularities [49], easier inflation [50, 51], correspondence between LQC and braneworld cosmology [52], and so on. However, as shown in e.g. [53], the first type of modification to Friedmann equation suffers from gauge dependence which can not be cured and thus lead to unphysical effects. In a recent paper [64], Magueijo and Singh provided very sharp results to show that it is only for the closed model that such modifications can be sensible and for flat models they make no sense.

The second type of modification to Friedmann equation is discovered very recently [44, 53, 54], which essentially encodes the discrete quantum geometric nature of spacetime. The corresponding effective modified Friedmann equation in a flat universe is given by [44, 53, 54, 57, 58, 59, 60]

$$
H^{2}=\frac{\kappa^{2} \rho}{3}\left(1-\frac{\rho}{\rho_{c}}\right),
$$


where $H \equiv \dot{a} / a$ is the Hubble parameter; a dot denotes the derivative with respect to cosmic time $t$, and $a$ is the scale factor; $\rho$ is the total energy density; $\kappa^{2} \equiv 8 \pi G$; and the critical density reads

$$
\rho_{c} \equiv \frac{\sqrt{3}}{16 \pi^{2} \gamma^{3} G^{2} \hbar},
$$

where $\gamma$ is the dimensionless Barbero-Immirzi parameter (it is suggested that $\gamma \simeq 0.2375$ by the black hole thermodynamics in LQG [56]). Differentiating Eq. (1) and using the energy conservation equation

$$
\dot{\rho}+3 H(\rho+p)=0,
$$

one obtain the effective modified Raychaudhuri equation [54, [57, [58, [59, 60]

$$
\dot{H}=-\frac{\kappa^{2}}{2}(\rho+p)\left(1-2 \frac{\rho}{\rho_{c}}\right),
$$

where $p$ is the total pressure. Actually, as shown in [64], the effective modified Raychaudhuri equation can be derived directly by using the Hamilton's equations in LQC, without assuming the energy conservation equation. It is easy to check that only two of Eqs. (11), (3) and (4) are independent of each other, and the third one can be derived from these two. By using the second type of modification to Friedmann equation, the physically appealing features of the first type are retained [44], for instance, resolution of big bang singularity [44, 53], avoidance of big rip and other singularities [57, 58, 59], inflation in LQC [60], correspondence between LQC and braneworld cosmology [54], and so on.

For the universe with a large scale factor, the first type of modification to the effective Friedmann equation can be neglected and only the second type of modification is important [54], while the matter Hamiltonian $\mathcal{H}_{M}$ and the corresponding expressions for energy density and pressure retain the same classical forms [44, 53, 54, 55, 57, 58, 59]. This is the particular case which we will consider here.

In the present work, we will investigate the universe dominated by quintom or hessence in LQC. Following [54, 57, 58, 59], we use the method of dynamical system [61]. After a brief review of quintom and hessence energies in Sec. [II we consider the dynamics of quintom and hessence in Sec. [III and Sec. [IV respectively. Interestingly enough, we find that there are some stable attractors in these two cases. In the case of quintom, all stable attractors have the feature of decelerated expansion. In the case of hessence, most of stable attractors have the feature of decelerated expansion while one stable attractor can have decelerated or accelerated expansion depend on the model parameter. In all cases, the EoS of all stable attractors are larger than -1 and there is no singularity in the finite future. These results are different from the dynamics of phantom in LQC [58, 59], or the ones of phantom, quintom and hessence in classical Einstein gravity [19, 20, 23, 24, 63].

\section{QUINTOM AND HESSENCE ENERGIES}

\section{A. Quintom energy}

Phenomenologically, one may consider the Lagrangian density for quintom [10, 23, 24]

$$
\mathcal{L}_{q}=\frac{1}{2}\left(\partial_{\mu} \phi_{1}\right)^{2}-\frac{1}{2}\left(\partial_{\mu} \phi_{2}\right)^{2}-V\left(\phi_{1}, \phi_{2}\right),
$$

where $\phi_{1}$ and $\phi_{2}$ are two real scalar fields and play the roles of quintessence and phantom respectively. Considering a spatially flat Friedmann-Robertson-Walker (FRW) universe and assuming the scalar fields $\phi_{1}$ and $\phi_{2}$ are homogeneous, one obtains the effective pressure and energy density for the quintom, i.e.

$$
p=\frac{1}{2} \dot{\phi}_{1}^{2}-\frac{1}{2} \dot{\phi}_{2}^{2}-V\left(\phi_{1}, \phi_{2}\right), \quad \rho=\frac{1}{2} \dot{\phi}_{1}^{2}-\frac{1}{2} \dot{\phi}_{2}^{2}+V\left(\phi_{1}, \phi_{2}\right),
$$


respectively. The corresponding effective EoS is given by

$$
w \equiv \frac{p}{\rho}=\frac{\dot{\phi}_{1}^{2}-\dot{\phi}_{2}^{2}-2 V\left(\phi_{1}, \phi_{2}\right)}{\dot{\phi}_{1}^{2}-\dot{\phi}_{2}^{2}+2 V\left(\phi_{1}, \phi_{2}\right)} .
$$

It is easy to see that $w \geq-1$ when $\dot{\phi}_{1}^{2} \geq \dot{\phi}_{2}^{2}$ while $w<-1$ when $\dot{\phi}_{1}^{2}<\dot{\phi}_{2}^{2}$. We consider the simplest quintom whose $V\left(\phi_{1}, \phi_{2}\right)=V_{1}\left(\phi_{1}\right)+V_{2}\left(\phi_{2}\right)$ in the present work. In this case, the equations of motion for $\phi_{1}$ and $\phi_{2}$ are given by

$$
\begin{aligned}
& \ddot{\phi}_{1}+3 H \dot{\phi}_{1}+\frac{d V_{1}}{d \phi_{1}}=0, \\
& \ddot{\phi}_{2}+3 H \dot{\phi}_{2}-\frac{d V_{2}}{d \phi_{2}}=0 .
\end{aligned}
$$

\section{B. Hessence energy}

Following [18, 19, 30], we consider a non-canonical complex scalar field, namely the hessence,

$$
\Phi=\phi_{1}+i \phi_{2},
$$

with a Lagrangian density

$$
\mathcal{L}_{h}=\frac{1}{4}\left[\left(\partial_{\mu} \Phi\right)^{2}+\left(\partial_{\mu} \Phi^{*}\right)^{2}\right]-U\left(\Phi^{2}+\Phi^{* 2}\right)=\frac{1}{2}\left[\left(\partial_{\mu} \phi\right)^{2}-\phi^{2}\left(\partial_{\mu} \theta\right)^{2}\right]-V(\phi),
$$

where we have introduced two new variables $(\phi, \theta)$ to describe the hessence, i.e.

$$
\phi_{1}=\phi \cosh \theta, \quad \phi_{2}=\phi \sinh \theta,
$$

which are defined by

$$
\phi^{2}=\phi_{1}^{2}-\phi_{2}^{2}, \quad \operatorname{coth} \theta=\frac{\phi_{1}}{\phi_{2}} .
$$

In fact, it is easy to see that in terms of $\phi_{1}$ and $\phi_{2}$, the hessence can be regarded as a special case of quintom with general $V\left(\phi_{1}, \phi_{2}\right)$. Considering a spatially flat FRW universe with scale factor $a(t)$ and assuming $\phi$ and $\theta$ are homogeneous, from Eq. (11) we obtain the equations of motion for $\phi$ and $\theta$,

$$
\begin{aligned}
& \ddot{\phi}+3 H \dot{\phi}+\phi \dot{\theta}^{2}+\frac{d V}{d \phi}=0, \\
& \phi^{2} \ddot{\theta}+\left(2 \phi \dot{\phi}+3 H \phi^{2}\right) \dot{\theta}=0 .
\end{aligned}
$$

The pressure and energy density of the hessence are

$$
p=\frac{1}{2}\left(\dot{\phi}^{2}-\phi^{2} \dot{\theta}^{2}\right)-V(\phi), \quad \rho=\frac{1}{2}\left(\dot{\phi}^{2}-\phi^{2} \dot{\theta}^{2}\right)+V(\phi),
$$

respectively. Eq. (15) implies

$$
Q=a^{3} \phi^{2} \dot{\theta}=\text { const. }
$$

which is associated with the total conserved charge within the physical volume due to the internal symmetry [18, 19]. It turns out that

$$
\dot{\theta}=\frac{Q}{a^{3} \phi^{2}}
$$


Substituting into Eqs. (14) and (16), they can be rewritten as

$$
\begin{gathered}
\ddot{\phi}+3 H \dot{\phi}+\frac{Q^{2}}{a^{6} \phi^{3}}+\frac{d V}{d \phi}=0 \\
p=\frac{1}{2} \dot{\phi}^{2}-\frac{Q^{2}}{2 a^{6} \phi^{2}}-V(\phi), \quad \rho=\frac{1}{2} \dot{\phi}^{2}-\frac{Q^{2}}{2 a^{6} \phi^{2}}+V(\phi) .
\end{gathered}
$$

Noting that the $\operatorname{EoS} w \equiv p / \rho$, it is easy to see that $w \geq-1$ when $\dot{\phi}^{2} \geq Q^{2} /\left(a^{6} \phi^{2}\right)$, while $w<-1$ when $\dot{\phi}^{2}<Q^{2} /\left(a^{6} \phi^{2}\right)$. We refer to the original papers [18, 19, 30] for more details.

\section{DYNAMICS OF QUINTOM ENERGY IN LQC}

In this section, we consider the universe dominated by quintom energy in LQC. Following [23, 24, 58, [59, 62], we introduce these five dimensionless variables

$$
x_{1} \equiv \frac{\kappa \dot{\phi}_{1}}{\sqrt{6} H}, \quad x_{2} \equiv \frac{\kappa \dot{\phi}_{2}}{\sqrt{6} H}, \quad y_{1} \equiv \frac{\kappa \sqrt{V_{1}}}{\sqrt{3} H}, \quad y_{2} \equiv \frac{\kappa \sqrt{V_{2}}}{\sqrt{3} H}, \quad z \equiv \frac{\rho}{\rho_{c}} .
$$

We introduce $z$ just for convenience. It is expected that $z$ is not independent, because of Eq. (6). In fact, in the case of the universe dominated by quintom energy in LQC, the effective modified Friedmann equation, namely Eq. (11), can be rewritten as

$$
\left(x_{1}^{2}-x_{2}^{2}+y_{1}^{2}+y_{2}^{2}\right)(1-z)=1 .
$$

Thus, one can explicitly express $z$ in terms of $x_{1}, x_{2}, y_{1}$ and $y_{2}$. Notice that $0 \leq z \leq 1$ is required by the positiveness of $\rho$ and $H^{2}$ in Eq. (11). In addition, by using Eq. (6), we recast Eq. (4) as

$$
-\frac{\dot{H}}{H^{2}}=3\left(x_{1}^{2}-x_{2}^{2}\right)(1-2 z),
$$

which will be used extensively. Also, Eq. (7) becomes

$$
w=\frac{x_{1}^{2}-x_{2}^{2}-y_{1}^{2}-y_{2}^{2}}{x_{1}^{2}-x_{2}^{2}+y_{1}^{2}+y_{2}^{2}}
$$

In this work, we consider the case of quintom with exponential potentials

$$
V_{1}\left(\phi_{1}\right)=V_{\phi_{1}} e^{-\lambda_{1} \kappa \phi_{1}}, \quad V_{2}\left(\phi_{2}\right)=V_{\phi_{2}} e^{-\lambda_{2} \kappa \phi_{2}},
$$

where $\lambda_{1}$ and $\lambda_{2}$ are dimensionless constants. Without loss of generality, we choose $\lambda_{1}$ and $\lambda_{2}$ to be positive, since we can make them positive through field redefinition $\phi_{1} \rightarrow-\phi_{1}, \phi_{2} \rightarrow-\phi_{2}$ if $\lambda_{1}$ and $\lambda_{2}$ are negative. By the help of Eqs. (22) - (25), (6) and (3), the equations of motion for $\phi_{1}$ and $\phi_{2}$, namely Eqs. (8) and (9), can be rewritten as an autonomous system

$$
\begin{aligned}
& x_{1}^{\prime}=3 x_{1}(A-1)+\sqrt{\frac{3}{2}} \lambda_{1} y_{1}^{2}, \\
& x_{2}^{\prime}=3 x_{2}(A-1)-\sqrt{\frac{3}{2}} \lambda_{2} y_{2}^{2}, \\
& y_{1}^{\prime}=3 y_{1}\left(A-\frac{\lambda_{1}}{\sqrt{6}} x_{1}\right), \\
& y_{2}^{\prime}=3 y_{2}\left(A-\frac{\lambda_{2}}{\sqrt{6}} x_{2}\right), \\
& z^{\prime}=-6 z\left(x_{1}^{2}-x_{2}^{2}\right)(1-z),
\end{aligned}
$$


where a prime denotes the derivative with respect to the so-called $e$-folding time $N \equiv \ln a$, and

$$
A \equiv\left(x_{1}^{2}-x_{2}^{2}\right)(1-2 z)=\left(x_{1}^{2}-x_{2}^{2}\right)\left[2\left(x_{1}^{2}-x_{2}^{2}+y_{1}^{2}+y_{2}^{2}\right)^{-1}-1\right],
$$

in which we have used Eq. (22). We can obtain the critical points $\left(\bar{x}_{1}, \bar{x}_{2}, \bar{y}_{1}, \bar{y}_{2}, \bar{z}\right)$ of the autonomous system Eqs. (26) - (30) by imposing the conditions $\bar{x}_{1}^{\prime}=\bar{x}_{2}^{\prime}=\bar{y}_{1}^{\prime}=\bar{y}_{2}^{\prime}=\bar{z}^{\prime}=0$. Of course, they are subject to the Friedmann constraint Eq. (22), namely $\left(\bar{x}_{1}^{2}-\bar{x}_{2}^{2}+\bar{y}_{1}^{2}+\bar{y}_{2}^{2}\right)(1-\bar{z})=1$. We present the critical points and their existence conditions in Table \. In fact, there are other four critical points with

$$
\bar{x}_{1}=\frac{\lambda_{1} \lambda_{2}^{2}}{\sqrt{6}\left(\lambda_{2}^{2}-\lambda_{1}^{2}\right)}, \quad \bar{x}_{2}=\frac{\lambda_{1}^{2} \lambda_{2}}{\sqrt{6}\left(\lambda_{2}^{2}-\lambda_{1}^{2}\right)}, \quad \bar{y}_{1}= \pm \lambda_{2} \sqrt{r_{q}}, \quad \bar{y}_{2}= \pm \lambda_{1} \sqrt{-r_{q}}, \quad \bar{z}=0,
$$

where

$$
r_{q} \equiv \frac{6 \lambda_{2}^{2}-\lambda_{1}^{2}\left(6+\lambda_{2}^{2}\right)}{6\left(\lambda_{2}^{2}-\lambda_{1}^{2}\right)^{2}} .
$$

\begin{tabular}{|c|c|c|}
\hline Label & Critical Point $\left(\bar{x}_{1}, \bar{x}_{2}, \bar{y}_{1}, \bar{y}_{2}, \bar{z}\right)$ & Existence \\
\hline Q1p & $\bar{x}_{1}^{2} \geq 1, \sqrt{\bar{x}_{1}^{2}-1}, \quad 0, \quad 0,0$ & $\bar{x}_{1}^{2} \geq 1$ \\
\hline Q1m & $\bar{x}_{1}^{2} \geq 1, \quad-\sqrt{\bar{x}_{1}^{2}-1}, \quad 0, \quad 0, \quad 0$ & $\bar{x}_{1}^{2} \geq 1$ \\
\hline Q2p & $\frac{\sqrt{6}}{\lambda_{1}}, \sqrt{\frac{6}{\lambda_{1}^{2}}-1}, 0,0, \quad 0$ & $\lambda_{1} \leq \sqrt{6}$ \\
\hline Q2m & $\frac{\sqrt{6}}{\lambda_{1}},-\sqrt{\frac{6}{\lambda_{1}^{2}}-1}, 0,0,0$ & $\lambda_{1} \leq \sqrt{6}$ \\
\hline Q3 & $\frac{\lambda_{1}}{\sqrt{6}}, 0, \pm \sqrt{1-\frac{\lambda_{1}^{2}}{6}}, 0,0$ & $\lambda_{1} \leq \sqrt{6}$ \\
\hline Q4p & $\sqrt{1+\frac{6}{\lambda_{2}^{2}}}, \frac{\sqrt{6}}{\lambda_{2}}, \quad 0, \quad 0, \quad 0$ & always \\
\hline Q4m & $-\sqrt{1+\frac{6}{\lambda_{2}^{2}}}, \frac{\sqrt{6}}{\lambda_{2}}, 0,0,0$ & always \\
\hline Q5 & $0,-\frac{\lambda_{2}}{\sqrt{6}}, 0, \pm \sqrt{1+\frac{\lambda_{2}^{2}}{6}}, 0$ & always \\
\hline
\end{tabular}

However, the existence of $\bar{y}_{1}$ and $\bar{y}_{2}$ requires $r_{q}=0$. In this case, these four critical points reduce to Points (Q2p) or (Q4p).

TABLE I: Critical points for the autonomous system Eqs. (26) - (30) and their existence conditions.

To study the stability of the critical points for the autonomous system Eqs. (26) - (30), we substitute linear perturbations $x_{1} \rightarrow \bar{x}_{1}+\delta x_{1}, x_{2} \rightarrow \bar{x}_{2}+\delta x_{2}, y_{1} \rightarrow \bar{y}_{1}+\delta y_{1}, y_{2} \rightarrow \bar{y}_{2}+\delta y_{2}$, and $z \rightarrow \bar{z}+\delta z$ about the critical point $\left(\bar{x}_{1}, \bar{x}_{2}, \bar{y}_{1}, \bar{y}_{2}, \bar{z}\right)$ into the autonomous system Eqs. (26) - (30) and linearize them. Because of the Friedmann constraint (22), there are only four independent evolution equations, i.e.

$$
\begin{aligned}
& \delta x_{1}^{\prime}=3 \bar{x}_{1} \delta A+3(\bar{A}-1) \delta x_{1}+\sqrt{6} \lambda_{1} \bar{y}_{1} \delta y_{1}, \\
& \delta x_{2}^{\prime}=3 \bar{x}_{2} \delta A+3(\bar{A}-1) \delta x_{2}-\sqrt{6} \lambda_{2} \bar{y}_{2} \delta y_{2}, \\
& \delta y_{1}=3 \bar{y}_{1}\left(\delta A-\frac{\lambda_{1}}{\sqrt{6}} \delta x_{1}\right)+3\left(\bar{A}-\frac{\lambda_{1}}{\sqrt{6}} \bar{x}_{1}\right) \delta y_{1}, \\
& \delta y_{2}=3 \bar{y}_{2}\left(\delta A-\frac{\lambda_{2}}{\sqrt{6}} \delta x_{2}\right)+3\left(\bar{A}-\frac{\lambda_{2}}{\sqrt{6}} \bar{x}_{2}\right) \delta y_{2},
\end{aligned}
$$

where

$$
\begin{aligned}
\bar{A}= & \left(\bar{x}_{1}^{2}-\bar{x}_{2}^{2}\right)\left[2\left(\bar{x}_{1}^{2}-\bar{x}_{2}^{2}+\bar{y}_{1}^{2}+\bar{y}_{2}^{2}\right)^{-1}-1\right], \\
\delta A= & -4\left(\bar{x}_{1}^{2}-\bar{x}_{2}^{2}\right)\left(\bar{x}_{1}^{2}-\bar{x}_{2}^{2}+\bar{y}_{1}^{2}+\bar{y}_{2}^{2}\right)^{-2}\left(\bar{x}_{1} \delta x_{1}-\bar{x}_{2} \delta x_{2}+\bar{y}_{1} \delta y_{1}+\bar{y}_{2} \delta y_{2}\right) \\
& +2\left[2\left(\bar{x}_{1}^{2}-\bar{x}_{2}^{2}+\bar{y}_{1}^{2}+\bar{y}_{2}^{2}\right)^{-1}-1\right]\left(\bar{x}_{1} \delta x_{1}-\bar{x}_{2} \delta x_{2}\right) .
\end{aligned}
$$


The four eigenvalues of the coefficient matrix of Eqs. (32) - (35) determine the stability of the critical point. We present the corresponding eigenvalues for the critical points in Table III It is easy to see that Points (Q1m), (Q2m), (Q3), (Q4m) and (Q5) are unstable. Point (Q1p) exists and is stable under conditions $\bar{x}_{1}>1, \lambda_{1} \bar{x}_{1} \geq \sqrt{6}$ and $\lambda_{2} \sqrt{\bar{x}_{1}^{2}-1} \geq \sqrt{6}$. Point (Q2p) exists and is stable under conditions $\lambda_{1} \leq \sqrt{6}$ and $\lambda_{2} \sqrt{9 \lambda_{1}^{-2}-3 / 2} \geq 3$. Point (Q4p) exists and is stable under condition $\lambda_{1} \sqrt{9 \lambda_{2}^{-2}+3 / 2} \geq 3$.

The three stable attractors (Q1p), (Q2p) and (Q4p) have the common features $\bar{y}_{1}=\bar{y}_{2}=\bar{z}=0$ and $\bar{x}_{1}^{2}-\bar{x}_{2}^{2}=1$. From Eq. (24), we get the $\operatorname{EoS} w=1$, which implies that the quintom behave as a stiff fluid. From Eq. (23),$-\dot{H} / H^{2}=3$. Then, we find that $H=t^{-1} / 3$ (the integral constant can be set to zero by redefining the time). Thus, we obtain $a \propto t^{1 / 3}$. From Eq. (3) and $w=1$, we find that $\rho \propto a^{-6} \propto t^{-2}$. The universe undergoes decelerated expansion and there is no singularity in the finite future.

\begin{tabular}{l|c}
\hline \hline Point & Eigenvalues \\
\hline Q1p & $-6, \quad 0,3-\sqrt{\frac{3}{2}} \lambda_{1} \bar{x}_{1}, 3-\lambda_{2} \sqrt{\frac{3}{2}\left(\bar{x}_{1}^{2}-1\right)}$ \\
Q1m & $-6, \quad 0,3-\sqrt{\frac{3}{2}} \lambda_{1} \bar{x}_{1}, 3+\lambda_{2} \sqrt{\frac{3}{2}\left(\bar{x}_{1}^{2}-1\right)}$ \\
Q2p & $-6, \quad 0,0,3-\lambda_{2} \sqrt{9 \lambda_{1}^{-2}-\frac{3}{2}}$ \\
Q2m & $-6, \quad 0,0,3+\lambda_{2} \sqrt{9 \lambda_{1}^{-2}-\frac{3}{2}}$ \\
Q3 & $-\lambda_{1}^{2}, \quad \frac{\lambda_{1}^{2}}{2}, \quad \frac{1}{2}\left(\lambda_{1}^{2}-6\right), \frac{1}{2}\left(\lambda_{1}^{2}-6\right)$ \\
Q4p & $-6, \quad 0,0,3-\lambda_{1} \sqrt{9 \lambda_{2}^{-2}+\frac{3}{2}}$ \\
Q4m & $-6, \quad 0,0,3+\lambda_{1} \sqrt{9 \lambda_{2}^{-2}+\frac{3}{2}}$ \\
Q5 & $-\frac{\lambda_{2}^{2}}{2}, \lambda_{2}^{2}, \quad-\frac{1}{2}\left(\lambda_{2}^{2}+6\right),-\frac{1}{2}\left(\lambda_{2}^{2}+6\right)$ \\
\hline \hline
\end{tabular}

TABLE II: The corresponding eigenvalues for the critical points of the autonomous system Eqs. (26) - (30).

\section{DYNAMICS OF HESSENCE ENERGY IN LQC}

In this section, we consider the universe dominated by hessence energy in LQC. Similar to the case of quintom, following [19, 20, 58, 59, 62], we introduce these five dimensionless variables

$$
x \equiv \frac{\kappa \dot{\phi}}{\sqrt{6} H}, \quad y \equiv \frac{\kappa \sqrt{V}}{\sqrt{3} H}, \quad z \equiv \frac{\rho}{\rho_{c}}, \quad u \equiv \frac{\sqrt{6}}{\kappa \phi}, \quad v \equiv \frac{\kappa}{\sqrt{6} H} \frac{Q}{a^{3} \phi} .
$$

Again, we introduce $z$ just for convenience, since it is expected that $z$ is not independent due to Eq. (20). In fact, in the case of the universe dominated by hessence energy in LQC, the effective modified Friedmann equation, namely Eq. (11), can be rewritten as

$$
\left(x^{2}+y^{2}-v^{2}\right)(1-z)=1,
$$

which can be used to explicitly express $z$ in terms of $x, y$ and $v$. Notice that $0 \leq z \leq 1$ is required by the positiveness of $\rho$ and $H^{2}$ in Eq. (1). By using Eq. (20), the effective modified Raychaudhuri equation, namely Eq. (4), can be rewritten as

$$
-\frac{\dot{H}}{H^{2}}=3\left(x^{2}-v^{2}\right)(1-2 z)
$$

From Eq. (20), the EoS of hessence is given by

$$
w \equiv \frac{p}{\rho}=\frac{x^{2}-v^{2}-y^{2}}{x^{2}-v^{2}+y^{2}} .
$$


In this work, we consider the case of hessence with exponential potential

$$
V(\phi)=V_{\phi} e^{-\lambda \kappa \phi}
$$

where $\lambda$ is a dimensionless constant. Without loss of generality, we choose $\lambda$ to be positive, since we can make it positive through field redefinition $\phi \rightarrow-\phi$ if $\lambda$ is negative. By the help of Eqs. (39) - (42), (20) and (3), the equation of motion for $\phi$, namely Eq. (19), can be rewritten as an autonomous system

$$
\begin{aligned}
x^{\prime} & =3 x(B-1)-u v^{2}+\sqrt{\frac{3}{2}} \lambda y^{2}, \\
y^{\prime} & =3 y\left(B-\frac{\lambda}{\sqrt{6}} x\right), \\
z^{\prime} & =-6 z\left(x^{2}-v^{2}\right)(1-z), \\
u^{\prime} & =-x u^{2} \\
v^{\prime} & =3 v\left(B-1-\frac{1}{3} x u\right),
\end{aligned}
$$

where

$$
B \equiv\left(x^{2}-v^{2}\right)(1-2 z)=\left(x^{2}-v^{2}\right)\left[2\left(x^{2}+y^{2}-v^{2}\right)^{-1}-1\right],
$$

in which we have used Eq. (39). We can obtain the critical points $(\bar{x}, \bar{y}, \bar{z}, \bar{u}, \bar{v})$ of the autonomous system Eqs. (43) - (47) by imposing the conditions $\bar{x}^{\prime}=\bar{y}^{\prime}=\bar{z}^{\prime}=\bar{u}^{\prime}=\bar{v}^{\prime}=0$. Of course, they are subject to the Friedmann constraint Eq. (39), namely $\left(\bar{x}^{2}+\bar{y}^{2}-\bar{v}^{2}\right)(1-\bar{z})=1$. We present the critical points and their existence conditions in Table III.

\begin{tabular}{l|c|c}
\hline \hline Label & Critical Point $(\bar{x}, \bar{y}, \bar{z}, \bar{u}, \bar{v})$ & Existence \\
\hline H1 & $\bar{x}^{2} \geq 1,0,0,0, \pm \sqrt{x^{2}-1}$ & $\bar{x}^{2} \geq 1$ \\
H2p & $1,0,0,0,0$ & always \\
H2m & $-1,0,0,0,0$ & always \\
H3 & $\frac{\sqrt{6}}{\lambda}, 0,0,0, \pm \sqrt{\frac{6}{\lambda^{2}}-1}$ & $\lambda \leq \sqrt{6}$ \\
H4 & $\frac{\lambda}{\sqrt{6}}, \pm \sqrt{1-\frac{\lambda^{2}}{6}}, 0,0,0$ & $\lambda \leq \sqrt{6}$ \\
\hline \hline
\end{tabular}

TABLE III: Critical points for the autonomous system Eqs. (43) - (47) and their existence conditions.

To study the stability of the critical points for the autonomous system Eqs. (43) - (47), we substitute linear perturbations $x \rightarrow \bar{x}+\delta x, y \rightarrow \bar{y}+\delta y, z \rightarrow \bar{z}+\delta z, u \rightarrow \bar{u}+\delta u$, and $v \rightarrow \bar{v}+\delta v$ about the critical point $(\bar{x}, \bar{y}, \bar{z}, \bar{u}, \bar{v})$ into the autonomous system Eqs. (43) - (47) and linearize them. Because of the Friedmann constraint (39), there are only four independent evolution equations, i.e.

$$
\begin{aligned}
\delta x^{\prime} & =3 \bar{x} \delta B+3(\bar{B}-1) \delta x-2 \bar{u} \bar{v} \delta v-\bar{v}^{2} \delta u+\sqrt{6} \lambda \bar{y} \delta y \\
\delta y^{\prime} & =3 \bar{y}\left(\delta B-\frac{\lambda}{\sqrt{6}} \delta x\right)+3\left(\bar{B}-\frac{\lambda}{\sqrt{6}} \bar{x}\right) \delta y \\
\delta u^{\prime} & =-2 \bar{x} \bar{u} \delta u-\bar{u}^{2} \delta x \\
\delta v^{\prime} & =3 \bar{v}\left[\delta B-\frac{1}{3}(\bar{x} \delta u+\bar{u} \delta x)\right]+3\left(\bar{B}-1-\frac{1}{3} \bar{x} \bar{u}\right) \delta v,
\end{aligned}
$$

where

$$
\begin{aligned}
\bar{B}= & \left(\bar{x}^{2}-\bar{v}^{2}\right)\left[2\left(\bar{x}^{2}+\bar{y}^{2}-\bar{v}^{2}\right)^{-1}-1\right] \\
\delta B= & -4\left(\bar{x}^{2}-\bar{v}^{2}\right)\left(\bar{x}^{2}+\bar{y}^{2}-\bar{v}^{2}\right)^{-2}(\bar{x} \delta x+\bar{y} \delta y-\bar{v} \delta v) \\
& +2\left[2\left(\bar{x}^{2}+\bar{y}^{2}-\bar{v}^{2}\right)^{-1}-1\right](\bar{x} \delta x-\bar{v} \delta v) .
\end{aligned}
$$


The four eigenvalues of the coefficient matrix of Eqs. (49) - (52) determine the stability of the critical point. We present the corresponding eigenvalues for the critical points in Table IV] It is easy to see that Point (H1) exists and is stable under conditions $\bar{x} \geq 1$ and $\lambda \bar{x} \geq \sqrt{6}$; Point (H2p) is stable under condition $\lambda \geq \sqrt{6}$; Point (H2m) is unstable; Points (H3) and (H4) are stable when they exist under condition $\lambda \leq \sqrt{6}$.

\begin{tabular}{l|c}
\hline \hline Point & Eigenvalues \\
\hline H1 & $-6, \quad 0,0,3-\sqrt{\frac{3}{2} \lambda} \bar{x}$ \\
H2p & $-6, \quad 0,0,3-\sqrt{\frac{3}{2}} \lambda$ \\
H2m & $-6, \quad 0,0,3+\sqrt{\frac{3}{2}} \lambda$ \\
H3 & $-6,0,0,0$ \\
H4 & $0,-\lambda^{2}, \quad \frac{1}{2}\left(\lambda^{2}-6\right), \frac{1}{2}\left(\lambda^{2}-6\right)$ \\
\hline \hline
\end{tabular}

TABLE IV: The corresponding eigenvalues for the critical points of the autonomous system Eqs. (43) - (47).

The stable attractors (H1), (H2p) and (H3) have the common features $\bar{y}=\bar{z}=\bar{u}=0$ and $\bar{x}^{2}-\bar{v}^{2}=1$. From Eq. (41), it is easy to find that the $\operatorname{EoS} w=1$, which implies that the hessence behave as a stiff fluid. From Eq. (40), $-\dot{H} / H^{2}=3$. Then, we find that $H=t^{-1} / 3$ (the integral constant can be set to zero by redefining the time). Thus, we obtain $a \propto t^{1 / 3}$. From Eq. (3) and $w=1$, we find that $\rho \propto a^{-6} \propto t^{-2}$. The universe undergoes decelerated expansion and there is no singularity in the finite future.

The stable attractor (H4) is slightly different from other three stable attractors. From Eq. (41), we get the $\operatorname{EoS} w=-1+\lambda^{2} / 3 \geq-1$. Note that $w \geq-1 / 3$ for $\sqrt{2} \leq \lambda \leq \sqrt{6}$, while $w<-1 / 3$ for $\lambda<\sqrt{2}$. From Eq. (40), $-\dot{H} / H^{2}=\lambda^{2} / 2$. Then, we find that $H=2 t^{-1} / \lambda^{2}$ (the integral constant can be set to zero by redefining the time). Thus, we obtain $a \propto t^{2 / \lambda^{2}}$. From Eq. (3) and $w=-1+\lambda^{2} / 3$, we find that $\rho \propto a^{-\lambda^{2}} \propto t^{-2}$. The universe experiences decelerated expansion for $\sqrt{2} \leq \lambda \leq \sqrt{6}$, or accelerated expansion for $\lambda<\sqrt{2}$. However, the universe cannot undergo super-accelerated expansion $(\dot{H}>0)$ for any $\lambda$. Therefore, there is no singularity in the finite future for any case.

\section{CONCLUSION}

In the framework of classical Einstein gravity, the dynamics of phantom, quintom and hessence have been studied in literature [19, 20, 23, 24, 63]. In the case of phantom, the universe will end in a big rip singularity [63]. In the case of quintom without direct couping between $\phi_{1}$ and $\phi_{2}$ [23], or with a special interaction between $\phi_{1}$ and $\phi_{2}$ through $V_{i n t} \sim\left[V_{1}\left(\phi_{1}\right) V_{2}\left(\phi_{2}\right)\right]^{1 / 2}$ 24 , the phantom-dominated solution is the unique attractor and the big rip is inevitable. In the case of hessence [19, 20], however, the big rip can be avoided, while its attractors are slightly different from the ones of the present work.

In the framework of LQC, the dynamics of phantom has also been studied [58, 59]. It is found that there is no stable attractor in this case. Therefore, the phase trajectory is very sensitive to initial conditions. However, the big rip can be avoided and the universe finally enters oscillatory regime. This is mainly due to the quantum correction to Friedmann equation.

In the present work, we investigate the universe dominated by quintom or hessence energies in Loop Quantum Cosmology (LQC). Interestingly enough, we find that there are some stable attractors in these two cases. In the case of quintom, all stable attractors have the feature of decelerated expansion. In the case of hessence, most of stable attractors have the feature of decelerated expansion while one stable attractor can have decelerated or accelerated expansion depend on the model parameter. In all cases, the equation-of-state parameter $(\mathrm{EoS})$ of all stable attractors are larger than -1 and there is no singularity in the finite future. These results are different from the dynamics of phantom in LQC, or the ones of phantom, quintom and hessence in classical Einstein gravity. 


\section{ACKNOWLEDGMENTS}

We are grateful to Professor Rong-Gen Cai for helpful discussions. We also thank Xin Zhang, ZongKuan Guo, Hui Li, and Sumin Tang, Shi-Chao Tang, Jian Hu, Yue Shen, Xin Liu, Lin Lin, Jing Jin, Wei-Ke Xiao, Feng-Yun Rao, Nan Liang, Rong-Jia Yang, Jian Wang, Yuan Liu for kind help and discussions. We acknowledge partial funding support from China Postdoctoral Science Foundation, and by the Ministry of Education of China, Directional Research Project of the Chinese Academy of Sciences under project No. KJCX2-YW-T03, and by the National Natural Science Foundation of China under project No. 10521001.

[1] P. J. E. Peebles and B. Ratra, Rev. Mod. Phys. 75, 559 (2003) astro-ph/0207347;

T. Padmanabhan, Phys. Rept. 380, 235 (2003) hep-th/0212290;

S. M. Carroll, astro-ph/0310342

R. Bean, S. Carroll and M. Trodden, astro-ph/0510059

V. Sahni and A. A. Starobinsky, Int. J. Mod. Phys. D 9, 373 (2000) astro-ph/9904398;

S. M. Carroll, Living Rev. Rel. 4, 1 (2001) astro-ph/0004075;

T. Padmanabhan, Curr. Sci. 88, 1057 (2005) astro-ph/0411044;

S. Weinberg, Rev. Mod. Phys. 61, 1 (1989);

S. Nobbenhuis, Found. Phys. 36, 613 (2006) gr-qc/0411093;

E. J. Copeland, M. Sami and S. Tsujikawa, Int. J. Mod. Phys. D 15, 1753 (2006) hep-th/0603057;

A. Albrecht et al., astro-ph/0609591.

R. Trotta and R. Bower, astro-ph/0607066.

[2] A. G. Riess et al. [Supernova Search Team Collaboration], Astron. J. 116, 1009 (1998) astro-ph/9805201;

S. Perlmutter et al. [Supernova Cosmology Project Collaboration], Astrophys. J. 517, 565 (1999) astro-ph/9812133;

J. L. Tonry et al. [Supernova Search Team Collaboration], Astrophys. J. 594, 1 (2003) astro-ph/0305008;

R. A. Knop et al., [Supernova Cosmology Project Collaboration], Astrophys. J. 598, 102 (2003) astro-ph/0309368;

A. G. Riess et al. [Supernova Search Team Collaboration], Astrophys. J. 607, 665 (2004) astro-ph/0402512.

[3] P. Astier et al. [SNLS Collaboration], Astron. Astrophys. 447, 31 (2006) astro-ph/0510447;

J. D. Neill et al. [SNLS Collaboration], astro-ph/0605148

[4] C. L. Bennett et al. [WMAP Collaboration], Astrophys. J. Suppl. 148, 1 (2003) astro-ph/0302207];

D. N. Spergel et al. [WMAP Collaboration], Astrophys. J. Suppl. 148175 (2003) astro-ph/0302209];

D. N. Spergel et al. [WMAP Collaboration], astro-ph/0603449;

L. Page et al. [WMAP Collaboration], astro-ph/0603450

G. Hinshaw et al. [WMAP Collaboration], astro-ph/0603451.

N. Jarosik et al. [WMAP Collaboration], astro-ph/0603452.

[5] M. Tegmark et al. [SDSS Collaboration], Phys. Rev. D 69, 103501 (2004) astro-ph/0310723;

M. Tegmark et al. [SDSS Collaboration], Astrophys. J. 606, 702 (2004) astro-ph/0310725];

U. Seljak et al., Phys. Rev. D 71, 103515 (2005) astro-ph/0407372; 
J. K. Adelman-McCarthy et al. [SDSS Collaboration], Astrophys. J. Suppl. 162, 38 (2006) astro-ph/0507711;

K. Abazajian et al. [SDSS Collaboration], astro-ph/0410239 astro-ph/0403325, astro-ph/0305492,

M. Tegmark et al. [SDSS Collaboration], Phys. Rev. D 74, 123507 (2006) astro-ph/0608632.

[6] S. W. Allen, R. W. Schmidt, H. Ebeling, A. C. Fabian and L. van Speybroeck, Mon. Not. Roy. Astron. Soc. 353, 457 (2004) astro-ph/0405340.

[7] A. G. Riess et al. [Supernova Search Team Collaboration], astro-ph/0611572

The numerical data of the full sample are available at

http://braeburn.pha.jhu.edu/ ariess/R06 or upon request to ariess@stsci.edu

[8] W. M. Wood-Vasey et al. [ESSENCE Collaboration], astro-ph/0701041;

G. Miknaitis et al. [ESSENCE Collaboration], astro-ph/0701043.

[9] D. Huterer and A. Cooray, Phys. Rev. D 71, 023506 (2005) astro-ph/0404062.

[10] B. Feng, X. L. Wang and X. M. Zhang, Phys. Lett. B 607, 35 (2005) astro-ph/0404224.

[11] J. Q. Xia, G. B. Zhao, B. Feng, H. Li and X. M. Zhang, Phys. Rev. D 73, 063521 (2006) astro-ph/0511625;

J. Q. Xia, G. B. Zhao, B. Feng and X. M. Zhang, JCAP 0609, 015 (2006) astro-ph/0603393;

G. B. Zhao, J. Q. Xia, B. Feng and X. M. Zhang, astro-ph/0603621;

J. Q. Xia, G. B. Zhao, H. Li, B. Feng and X. M. Zhang, Phys. Rev. D 74, 083521 (2006) astro-ph/0605366;

J. Q. Xia, G. B. Zhao and X. M. Zhang, Phys. Rev. D 75, 103505 (2007) astro-ph/0609463;

G. B. Zhao, J. Q. Xia, H. Li, C. Tao, J. M. Virey, Z. H. Zhu and X. M. Zhang, Phys. Lett. B 648, 8 (2007) astro-ph/0612728.

[12] Y. Wang and M. Tegmark, Phys. Rev. D 71, 103513 (2005) astro-ph/0501351.

[13] U. Alam, V. Sahni and A. A. Starobinsky, JCAP 0406, 008 (2004) astro-ph/0403687.

[14] B. A. Bassett, P. S. Corasaniti and M. Kunz, Astrophys. J. 617, L1 (2004) astro-ph/0407364;

A. Cabre, E. Gaztanaga, M. Manera, P. Fosalba and F. Castander, Mon. Not. Roy. Astron. Soc. Lett. 372, L23 (2006) astro-ph/0603690.

[15] S. Nesseris and L. Perivolaropoulos, Phys. Rev. D 70, 043531 (2004) astro-ph/0401556;

R. Lazkoz, S. Nesseris and L. Perivolaropoulos, JCAP 0511, 010 (2005) astro-ph/0503230.

[16] Y. Wang and P. Mukherjee, Astrophys. J. 650, 1 (2006) astro-ph/0604051.

[17] A. Upadhye, M. Ishak and P. J. Steinhardt, Phys. Rev. D 72, 063501 (2005) astro-ph/0411803.

[18] H. Wei, R. G. Cai and D. F. Zeng, Class. Quant. Grav. 22, 3189 (2005) hep-th/0501160.

[19] H. Wei and R. G. Cai, Phys. Rev. D 72, 123507 (2005) astro-ph/0509328.

[20] M. Alimohammadi and H. Mohseni Sadjadi, Phys. Rev. D 73, 083527 (2006) hep-th/0602268.

[21] W. Zhao and Y. Zhang, Phys. Rev. D 73, 123509 (2006) astro-ph/0604460.

[22] H. Wei and R. G. Cai, Phys. Lett. B 634, 9 (2006) astro-ph/0512018;

H. Wei and R. G. Cai, Phys. Rev. D 73, 083002 (2006) astro-ph/0603052.

[23] Z. K. Guo, Y. S. Piao, X. M. Zhang and Y. Z. Zhang, Phys. Lett. B 608, 177 (2005) astro-ph/0410654.

[24] X. F. Zhang, H. Li, Y. S. Piao and X. M. Zhang, Mod. Phys. Lett. A 21, 231 (2006) astro-ph/0501652.

[25] X. Zhang, Int. J. Mod. Phys. D 14, 1597 (2005) astro-ph/0504586;

Z. Chang, F. Q. Wu and X. Zhang, Phys. Lett. B 633, 14 (2006) astro-ph/0509531;

X. Zhang and F. Q. Wu, Phys. Rev. D 72, 043524 (2005) astro-ph/0506310;

X. Zhang, Phys. Rev. D 74, 103505 (2006) astro-ph/0609699. 
[26] H. S. Zhang and Z. H. Zhu, Phys. Rev. D 73, 043518 (2006) astro-ph/0509895;

H. S. Zhang and Z. H. Zhu, Phys. Rev. D 75, 023510 (2007) astro-ph/0611834;

H. S. Zhang and Z. H. Zhu, astro-ph/0703245;

H. S. Zhang and Z. H. Zhu, arXiv:0704.3121 [astro-ph].

[27] H. Wei and R. G. Cai, astro-ph/0607064.

[28] A. Vikman, Phys. Rev. D 71, 023515 (2005) astro-ph/0407107.

[29] H. Wei and S. N. Zhang, Phys. Lett. B 644, 7 (2007) astro-ph/0609597;

H. Wei and S. N. Zhang, arXiv:0704.3330 [astro-ph].

[30] H. Wei, N. N. Tang and S. N. Zhang, Phys. Rev. D 75, 043009 (2007) astro-ph/0612746.

[31] Z. K. Guo, Y. S. Piao, X. M. Zhang and Y. Z. Zhang, Phys. Rev. D 74, 127304 (2006) astro-ph/0608165; M. Z. Li, B. Feng and X. M. Zhang, JCAP 0512, 002 (2005) hep-ph/0503268;

X. F. Zhang and T. T. Qiu, Phys. Lett. B 642, 187 (2006) astro-ph/0603824;

Y. F. Cai, H. Li, Y. S. Piao and X. M. Zhang, Phys. Lett. B 646, 141 (2007) gr-qc/0609039;

Y. F. Cai, M. Z. Li, J. X. Lu, Y. S. Piao, T. T. Qiu and X. M. Zhang, hep-th/0701016,

Y. F. Cai, T. T. Qiu, Y. S. Piao, M. Z. Li and X. M. Zhang, arXiv:0704.1090 [gr-qc];

R. Lazkoz, G. Leon and I. Quiros, Phys. Lett. B 649, 103 (2007) astro-ph/0701353;

R. Lazkoz and G. Leon, Phys. Lett. B 638, 303 (2006) astro-ph/0602590;

M. R. Setare, Phys. Lett. B 641, 130 (2006) hep-th/0611165;

M. Alimohammadi and H. M. Sadjadi, Phys. Lett. B 648, 113 (2007) gr-qc/0608016;

H. Mohseni Sadjadi and M. Alimohammadi, Phys. Rev. D 74, 043506 (2006) gr-qc/0605143;

W. Wang, Y. X. Gui and Y. Shao, Chin. Phys. Lett. 23, 762 (2006);

P. X. Wu and H. W. Yu, Int. J. Mod. Phys. D 14, 1873 (2005) gr-qc/0509036.

[32] P. S. Apostolopoulos and N. Tetradis, Phys. Rev. D 74, 064021 (2006) hep-th/0604014.

[33] E. Elizalde, S. Nojiri and S. D. Odintsov, Phys. Rev. D 70, 043539 (2004) hep-th/0405034;

S. Nojiri, S. D. Odintsov and S. Tsujikawa, Phys. Rev. D 71, 063004 (2005) hep-th/0501025;

S. Nojiri and S. D. Odintsov, Gen. Rel. Grav. 38, 1285 (2006) hep-th/0506212;

S. Capozziello, S. Nojiri and S. D. Odintsov, Phys. Lett. B 632, 597 (2006) hep-th/0507182;

S. Nojiri and S. D. Odintsov, Phys. Rev. D 72, 023003 (2005) hep-th/0505215;

E. Elizalde, S. Nojiri, S. D. Odintsov and P. Wang, Phys. Rev. D 71, 103504 (2005) hep-th/0502082.

[34] E. O. Kahya and V. K. Onemli, gr-qc/0612026

T. Brunier, V. K. Onemli and R. P. Woodard, Class. Quant. Grav. 22, 59 (2005) gr-qc/0408080.

[35] I. Y. Aref'eva, A. S. Koshelev and S. Y. Vernov, Phys. Rev. D 72, 064017 (2005) astro-ph/0507067;

I. Y. Aref'eva and A. S. Koshelev, JHEP 0702, 041 (2007) hep-th/0605085;

I. Y. Aref'eva, AIP Conf. Proc. 826, 301 (2006) astro-ph/0410443;

I. Y. Aref'eva, L. V. Joukovskaya and S. Y. Vernov, hep-th/0701184

[36] G. B. Zhao, J. Q. Xia, M. Li, B. Feng and X. M. Zhang, Phys. Rev. D 72, 123515 (2005) astro-ph/0507482.

[37] M. Kunz and D. Sapone, Phys. Rev. D 74, 123503 (2006) astro-ph/0609040.

[38] C. Rovelli, Living Rev. Rel. 1, 1 (1998) gr-qc/9710008;

T. Thiemann, Lect. Notes Phys. 631, 41 (2003) gr-qc/0210094;

M. Bojowald, gr-qc/0505057. 
A. Corichi, J. Phys. Conf. Ser. 24, 1 (2005) gr-qc/0507038;

A. Perez, gr-qc/0409061

[39] A. Ashtekar and J. Lewandowski, Class. Quant. Grav. 21, R53 (2004) gr-qc/0404018;

A. Ashtekar, arXiv:0705.2222 [gr-qc].

[40] C. Rovelli, Quantum Gravity, Cambridge University Press, Cambridge (2004).

[41] A. Ashtekar, New J. Phys. 7, 198 (2005) gr-qc/0410054;

T. Thiemann, hep-th/0608210.

[42] M. Bojowald, Living Rev. Rel. 8, 11 (2005) gr-qc/0601085;

M. Bojowald, gr-qc/0505057.

[43] A. Ashtekar, M. Bojowald and J. Lewandowski, Adv. Theor. Math. Phys. 7, 233 (2003) gr-qc/0304074;

A. Ashtekar, gr-qc/0702030

[44] A. Ashtekar, AIP Conf. Proc. 861, 3 (2006) gr-qc/0605011.

[45] A. Ashtekar, T. Pawlowski and P. Singh, Phys. Rev. Lett. 96, 141301 (2006) gr-qc/0602086.

[46] M. Bojowald, P. Singh and A. Skirzewski, Phys. Rev. D 70, 124022 (2004) gr-qc/0408094.

[47] P. Singh and K. Vandersloot, Phys. Rev. D 72, 084004 (2005) gr-qc/0507029.

[48] A. Ashtekar, T. Pawlowski and P. Singh, Phys. Rev. D 73, 124038 (2006) gr-qc/0604013.

[49] P. Singh and A. Toporensky, Phys. Rev. D 69, 104008 (2004) gr-qc/0312110;

G. V. Vereshchagin, JCAP 0407, 013 (2004) gr-qc/0406108;

G. Date and G. M. Hossain, Phys. Rev. Lett. 94, 011302 (2005) gr-qc/0407074.

[50] M. Bojowald, Phys. Rev. Lett. 89, 261301 (2002) gr-qc/0206054;

M. Bojowald and K. Vandersloot, Phys. Rev. D 67, 124023 (2003) gr-qc/0303072;

M. Bojowald, J. E. Lidsey, D. J. Mulryne, P. Singh and R. Tavakol, Phys. Rev. D 70, 043530 (2004) gr-qc/0403106.

[51] S. Tsujikawa, P. Singh and R. Maartens, Class. Quant. Grav. 21, 5767 (2004) astro-ph/0311015;

J. E. Lidsey, D. J. Mulryne, N. J. Nunes and R. Tavakol, Phys. Rev. D 70, 063521 (2004) gr-qc/0406042];

D. J. Mulryne, N. J. Nunes, R. Tavakol and J. E. Lidsey, Int. J. Mod. Phys. A 20, 2347 (2005) gr-qc/0411125;

N. J. Nunes, Phys. Rev. D 72, 103510 (2005) astro-ph/0507683.

[52] E. J. Copeland, J. E. Lidsey and S. Mizuno, Phys. Rev. D 73, 043503 (2006) gr-qc/0510022.

[53] A. Ashtekar, T. Pawlowski and P. Singh, Phys. Rev. D 74, 084003 (2006) gr-qc/0607039.

[54] P. Singh, Phys. Rev. D 73, 063508 (2006) gr-qc/0603043.

[55] P. Singh, Class. Quant. Grav. 22, 4203 (2005) gr-qc/0502086].

[56] A. Ashtekar, J. Baez, A. Corichi and K. Krasnov, Phys. Rev. Lett. 80, 904 (1998) gr-qc/9710007;

M. Domagala and J. Lewandowski, Class. Quant. Grav. 21, 5233 (2004) gr-qc/0407051;

K. A. Meissner, Class. Quant. Grav. 21, 5245 (2004) gr-qc/0407052.

[57] M. Sami, P. Singh and S. Tsujikawa, Phys. Rev. D 74, 043514 (2006) gr-qc/0605113.

[58] D. Samart and B. Gumjudpai, arXiv:0704.3414 [gr-qc].

[59] T. Naskar and J. Ward, arXiv:0704.3606 [gr-qc].

[60] X. Zhang and Y. Ling, arXiv:0705.2656 [gr-qc].

[61] A. A. Coley, gr-qc/9910074

J. Wainwright and G. F. R. Ellis, Dynamical Systems in Cosmology, Cambridge University Press, Cambridge 
(1997);

A. A. Coley, Dynamical Systems and Cosmology, in Series: Astrophysics and Space Science Library, Vol. 291, Springer (2004).

[62] E. J. Copeland, A. R. Liddle and D. Wands, Phys. Rev. D 57, 4686 (1998) gr-qc/9711068.

[63] R. R. Caldwell, Phys. Lett. B 545, 23 (2002) astro-ph/9908168;

R. R. Caldwell, M. Kamionkowski and N. N. Weinberg, Phys. Rev. Lett. 91, 071301 (2003) astro-ph/0302506. [64] J. Magueijo and P. Singh, astro-ph/0703566 